04

\title{
Особенности получения изображений протяженных объектов, восстанавливаемых импульсными голограммами
}

\author{
() Н.Д. Ворзобова, П.П. Соколов \\ Университет ИТМО, \\ 197101 Санкт-Петербург, Россия \\ e-mail: vorzobova@mail.ifmo.ru
}

Поступила в редакцию 30.09.2020 г.

В окончательной редакции 25.11.2020 г.

Принята к публикации 30.11.2020 г.

Рассмотрены особенности размерной трансформации изображений протяженных по глубине объектов, восстанавливаемых пропускающими импульсными голограммами при различии спектральных и геометрических параметров записи голограмм и воспроизведения изображений. Предложен способ улучшения характеристик изображений, позволяющий снизить требования к выходным характеристикам импульсных лазеров.

Ключевые слова: импульсная голография, свойства голографических изображений протяженных объектов.

DOI: $10.21883 /$ OS.2021.03.50658.251-20

\section{Введение}

В настоящее время большой интерес проявляется к методам получения 3D изображений больших сцен [1-10]. Рассматриваемые в литературе методы основаны на различных принципах и эффектах, включающих стереоэффекты и эффекты параллакса $[1-4]$, различные способы синтеза изображений, в том числе с использованием цифровых технологий [5-10]. Однако эти методы технически сложны и сталкиваются с рядом проблем, связанных с соответствием формируемых изображений реальным объектам, особенно протяженным. В связи с этим определенные преимущества имеют аналоговые голографические методы, позволяющие получать изображения, в наибольшей степени отображающие свойства реальных объектов.

Особое место занимают методы, базирующиеся на голографической записи импульсным излучением, обеспечивающие возможность регистрировать нестационарные объекты. В предыдущие годы импульсная изобразительная голография развивалась преимущественно в направлении получения отражательных голограмм, позволяющих благодаря высокой спектральной селективности использовать для восстановления изображений источники белого света. Проблема прямой записи импульсным излучением отражательных голограмм в особомелкозернистых галогенсеребряных материалах, связанная с их низкой чувствительностью к излучению с малой длительностью импульсов [11], привела к разработке двухстадийных процессов [12], основанных на записи пропускающих голограмм импульсным излучением и последующем интерференционном копировании восстановленных изображений во встречных пучках непрерывным излучением. Преимуществом записи пропускающих голограмм на первой стадии является возможность использования крупнозернистых материалов, обладающих меньшей разрешающей способностью, но большей чувствительностью к импульсному излучению, обеспечивающей безопасность съемки живых объектов. Были разработаны процессы получения крупноформатных пропускающих голограмм с записью импульсным излучением в зеленой и красной областях спектра [13-17], которые используются преимущественно для последующего копирования.

Двухстадийные процессы получили применение в значительной степени благодаря возможности использования промышленных материалов ВРП, ФПР, ПФГ-01, ПФГ-03М („Славич“, Россия) и доступных источников белого света для воспроизведения изображений. Однако восстановление изображений источниками белого света вследствие их широкого спектрального состава не позволяет получать изображения объектов с большой протяженностью. В связи с этим определенные преимущества имеют процессы получения пропускающих импульсных голограмм, с восстановлением изображений в монохроматическом свете. Актуальность данной задачи определяется современным уровнем развития полупроводниковой техники и появлением дешевых малогабаритных источников монохроматического излучения высокой мощности, которые могут использоваться для воспроизведения изображений. При выборе длины волны монохроматического излучения необходимо учитывать возможность размерной трансформации изображений, которая применительно к протяженным по глубине объектам ранее детально не исследовалась и будет рассмотрена в данной работе.

Задача прямой записи высокоэффективных крупноформатных импульсных голограмм протяженных по глубине объектов для восстановления в монохроматическом свете выдвигает более высокие требования к характеристикам импульсного излучения по сравнению с двухстадийными процессами. При двухстадийных про- 
цессах некоторые недостатки импульсных голограмм, связанные с характеристиками импульсного излучения, в частности, недостаточная яркость изображений, могут компенсироваться на этапе копирования, а к глубине изображений объектов не предъявляется высоких требований вследствие ориентации на восстановление изображений в белом свете. Для получения пропускающих голограмм протяженных объектов с учетом расхода выходной энергии лазеров на освещение большой сцены, а также необходимости обеспечения высокой яркости изображений и качества голограмм требования к характеристикам импульсного излучения существенно возрастают, что неизбежно приводит к усложнению лазерных систем и их высокой стоимости. Поэтому актуальна задача снижения этих требований. В данной работе мы рассмотрим одну из возможностей уменьшения требований к характеристикам импульсного излучения.

\section{Размерная трансформация изображений протяженных объектов}

Рассмотрим, как различия в длинах волн излучения, используемого для записи голограммы, и восстанавливающего излучения, а также геометрических параметров схем записи голограммы и воспроизведения изображений будут влиять на размерные характеристики изображений протяженных объектов. Теоретический анализ проведен с использованием выражения [18], связывающего поперечное увеличение восстановленного изображения $V$ с положением записывающего $z_{r}$ и восстанавливающего $z_{c}$ источников, расстоянием до объекта $z_{1}$ при записи голограммы, а также с отношением длин волн восстанавливающего и записывающего излучения $\mu$ :

$$
V=\left(1+\frac{z_{1}}{\mu z_{c}}-\frac{z_{1}}{z_{r}}\right)^{-1},
$$

где $V$ - поперечное увеличение изображения, $z_{1}-$ расстояние от объекта до плоскости голограммы, $z_{r}-$ расстояние от опорного точечного источника до голограммы, $z_{c}$ - расстояние от восстанавливающего точечного источника до голограммы, $\mu$ - отношение длин волн восстанавливающего и записывающего излучений.

Расчеты проводились при изменении параметров в широких пределах. На рис. 1 приведены результаты для некоторых значений изменяемых параметров для заданных $z_{r}=0.8$ и $1.2 \mathrm{~m}$ при изменении положения восстанавливающего источника $z_{c}$ относительно положения записывающего источника на $0.2 \mathrm{~m}$ для трех длин волн восстанавливающего излучения: $0.53,0.65,0.44 \mu \mathrm{m}$. Длина волны записи голограммы $0.53 \mu \mathrm{m}$ соответствует разработанной технологии получения импульсных голограмм. Представленные ниже семейства кривых позволяют оценить тенденции изменения размерных характеристик восстановленных изображений протяженных по глубине объектов при изменении длин волн и

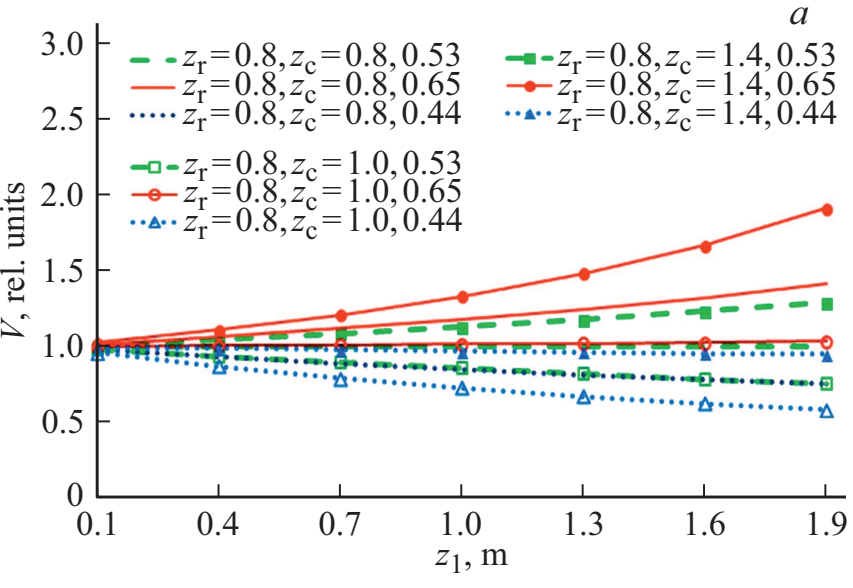

$b$

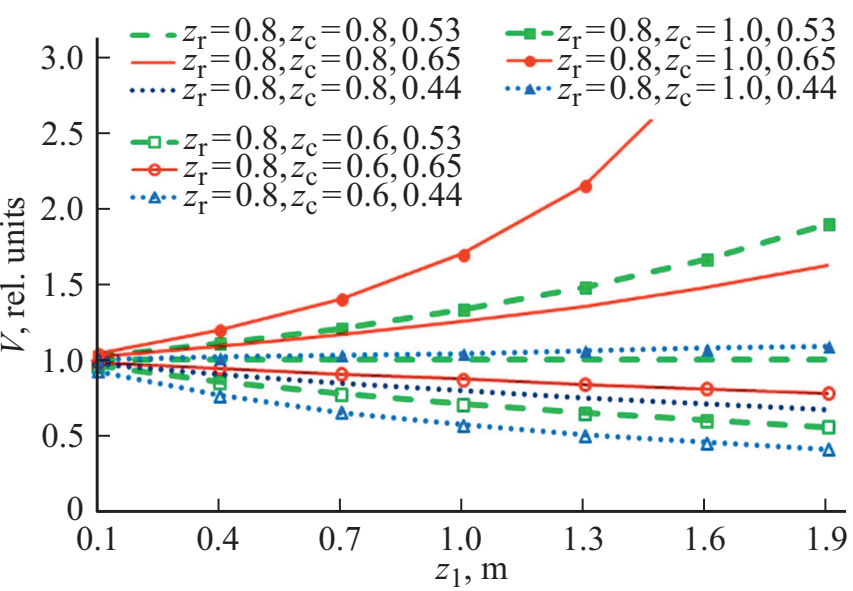

Рис. 1. Влияние длин волн и геометрических параметров схем записи голограмм и восстановления изображений на размерные характеристики изображений протяженных объектов. Длина волны записывающего излучения $0.53 \mu \mathrm{m}$. Расстояние $z_{r}$ от опорного источника при записи голограммы до плоскости голограммы $1.2(a)$ и $0.8 \mathrm{~m}(b)$.

геометрических параметров схем записи голограмм и восстановления изображений.

Можно видеть, что изменение длины волны восстанавливающего излучения относительно длины волны записывающего излучения может приводить к искажению перспективы - сжатию или растяжению, связанному с различием увеличений фрагментов объектов, расположенных на различных расстояниях от голограммы.

При различии условий записи голограммы и восстановления изображений наибольшие изменения в размеpax (в плоскости и по глубине изображения) наблюдаются при увеличении длины волны восстанавливающего излучения относительно длины волны излучения, используемого для записи голограммы, а также при уменьшении расстояния до опорного источника $\left(z_{r}\right)$. Размерные искажения увеличиваются при увеличении расстояния до объекта $\left(z_{1}\right)$. Компенсация размерных искажений возможна при уменьшении расстояния до 
восстанавливающего источника $\left(z_{c}\right)$ относительно расстояния до опорного источника $\left(z_{r}\right)$.

Уменьшение длины волны восстанавливающего излучения относительно длины волны записывающего излучения приводит к уменьшению размеров восстановленного изображения, а также уменьшению размерных искажений протяженных по глубине объектов. Размерные искажения уменьшаются с увеличением расстояния до объекта $\left(z_{1}\right)$. Компенсация размерных изменений возможна при увеличении $z_{c}$ относительно $z_{r}$.

Установленные тенденции были подтверждены экспериментально при наблюдении изображений протяженных объектов (с глубиной до $4 \mathrm{~m}$ ), восстановленных излучением с длинами волн $0.65,053$ и $0.44 \mu \mathrm{m}$ пропускающими голограммами, записанными на длине волны $0.53 \mu \mathrm{m}$.

Полученные закономерности следует учитывать при выборе источника монохроматического излучения и условий воспроизведения голографических изображений, а также проектировании осветителей.

\section{Снижение требований к характеристикам импульсного излучения}

Для получения высокоэффективных крупноформатных голограмм протяженных объектов требуется сочетание высокой выходной энергии импульсных лазеров, необходимой для освещения протяженной сцены с учетом отражательной способности объектов, высокой степени когерентности излучения для регистрации удаленных фрагментов, а также равномерности лазерного излучения. Следует отметить, что неравномерность излучения в опорном пучке может приводить к ухудшению качества голограмм - уменьшению дифракционной эффективности и увеличению светорассеяния, а также ухудшению визуального восприятия голографического изображения вследствие наличия в поле зрения наблюдателя поверхности голограммы с неравномерной оптической плотностью. Обеспечение перечисленных требований приводит к усложнению лазерных систем и, как следствие, их высокой стоимости. Кроме того, в процессе эксплуатации возможно ухудшение характеристик импульсного излучения - выходной энергии, равномерности, что приводит к необходимости замены дорогостоящих элементов импульсных лазеров.

В данном разделе мы рассмотрим способ, позволяющий снизить требования к характеристикам импульсного излучения. Способ основан на двухэкспозиционном экспонировании, приводящем к оптическому усилению скрытого изображения, образованного при записи импульсным излучением, посредством последующей засветки непрерывным излучением слабой интенсивности. Согласно имеющимся представлениям, в результате различий в длительностях электронной и ионной стадий формирования скрытого изображения в галогенсеребряных светочувствительных кристаллах [19] кратковременное действие интенсивного импульсного излучения приводит к формированию мелкодисперсного скрытого изображения. Постэкспозиционная низкоинтенсивная засветка обеспечивает достройку мелких центров скрытого изображения, сформированных при импульсной записи, до размеров, необходимых для их эффективного химического усиления.

Нами были проведены исследования влияния последующей засветки на голографические характеристики различных галогенсеребряных материалов - ПФГ-03 М, ПФГ-01, ВРП, ФПР, РНЦ „Курчатовский институт“ и др. с размерами светочувствительных кристаллов от 20 до $60 \mathrm{~nm}$ при различных процессах химической обработки. Эксперименты проводились при записи голограмм плоских волн излучением с длинами волн 0.53 и $0.69 \mu \mathrm{m}$ и длительностями импульсов 10 и $30 \mathrm{~ns}$. Дополнительная засветка проводилась рассеянным излучением лампы накаливания при изменении освещенности в диапазоне $10-1001 x$ и длительности освещения в диапазоне $3-50 \mathrm{~s}$. Дифракционная эффективность определялась отношением интенсивностей в первом порядке дифракции и падающего излучения. Светорассеяние оценивалось интенсивностью ореола рассеяния.

Для всех исследованных материалов было выявлено позитивное действие последующей засветки, а также критичность к освещенности, длительности засветки и процессу химической обработки. Применительно к промышленным материалам ПФГ-01, ВРП (ФПР) наилучшие результаты получены при освещенности $10-501 \mathrm{x}$ и количестве освещения 300 и $400 \mathrm{lxs}$ соответственно. На рис. 2 приведены результаты, полученные на промышленном материале ФПР при записи пропускающих голограмм излучением с длиной волны $0.53 \mu \mathrm{m}$ и длительностью импульса $10 \mathrm{~ns}$. Химическая обработка проводилась в проявителе CWC2 и отбеливателе PBU [20], обеспечивающих дифракционную эффективность до 80\%. Дополнительная засветка проводилась излучением лампы накаливания при освещенности $201 \mathrm{x}$ в течение $20 \mathrm{~s}$.

Можно видеть (кривые 1 и 3), что положение максимума светорассеяния сдвинуто относительно максимума дифракционной эффективности в сторону меньших экспозиций. При оптимальной экспозиции в опорном пучке, соответствующей максимуму дифракционной эффективности $\left(E_{1}\right)$, достигается низкий уровень светорассеяния. Однако уменьшение экспозиции $\left(E_{2}\right)$, а также наличие неравномерности интенсивности излучения в опорном пучке $(\Delta E)$ приводят к увеличению светорассеяния.

При дополнительной постэкспозиционной засветке наблюдается существенный сдвиг экспозиционных кривых дифракционной эффективности и светорассеяния в сторону малых экспозиций - в 8-10 раз (кривые 2 и 4) по оси плотности энергии. Уменьшается также величина максимума светорассеяния. В результате при экспонировании в области малых значений плотности 


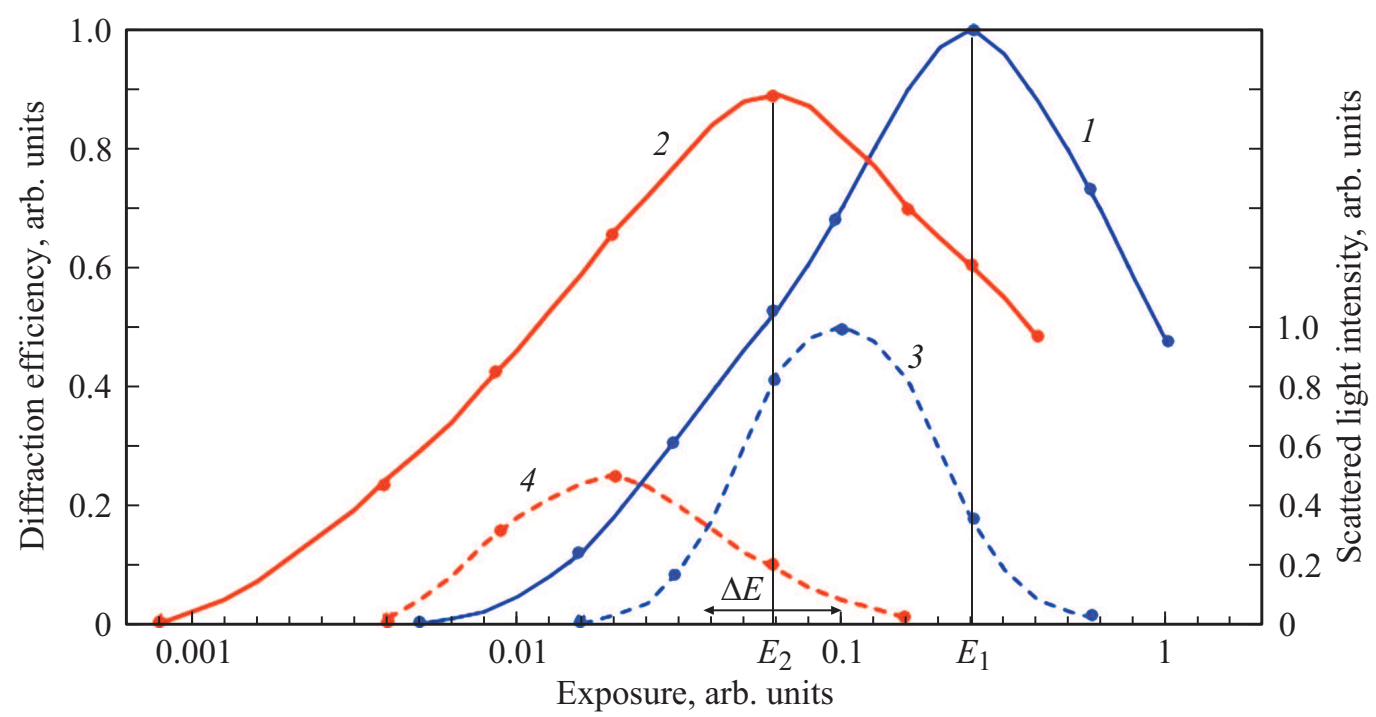

Рис. 2. Зависимость дифракционной эффективности $(1,2)$ и светорассеяния $(3,4)$ от экспозиции при импульсной голографической записи в материале ФПР без засветки $(1,3)$ и с дополнительной засветкой $(2,4)$.

энергии $\left(E_{2}\right)$, неизбежных при низкой выходной энергии лазеров, при больших размерах голограмм и размерах объектов дополнительная засветка позволяет существенно улучшить голографические характеристики увеличить дифракционную эффективность и уменьшить светорассеяние. Таким образом, снижаются требования к экспозиционным параметрам - рабочей экспозиции и равномерности интенсивности излучения в опорном пучке, а значит и выходным характеристикам импульсных лазеров. Кроме того, дополнительная засветка приводит к увеличению равномерности оптической плотности по полю голограммы, что улучшает зрительное восприятие восстановленного изображения.

Установленные закономерности и возможность улучшения качества изображений подтверждены при записи импульсных голограмм произвольных объектов при малой плотности энергии излучения в опорном пучке и его существенной неравномерности. Рассмотренный способ может являться простым и эффективным способом, позволяющим улучшить качество голограмм и снизить требования к характеристикам лазерного излучения.

\section{Коррекция цвета восстановленных изображений}

Голографические изображения, восстанавливаемые в монохроматическом свете, имеют цвет, соответствующий длине волны используемого источника. Для наилучшего физиологического восприятия предпочтительно использовать источники в желтой области спектра. Однако все еще существует проблема мощности, а также стоимости таких источников. При использовании более доступных и мощных полупроводниковых источников с длинами волн в зеленой, красной и синей областях спектра можно использовать аддитивную корректировку цвета. Идея заключается в дополнительной подсветке голограммы излучением с длиной волны, отличной от длины волны восстанавливающего излучения. При таком способе в глаз наблюдателя попадает некоторое количество света, рассеянного на материале голограммы и создающего аддитивный фон, на котором видно восстановленное изображение.

При выборе направления падения дополнительного подсвечивающего пучка необходимо учитывать возможность получения лишних изображений, возникающих в результате его дифракции на структуре голограммы. Исключение лишних изображений может быть достигнуто при введении угла между восстанавливающим и подсвечивающим пучками, определяемого угловой селективностью голограммы. Как показали наши эксперименты, для размера голограммы $30 \times 40 \mathrm{~cm}$ и углового размера объекта до $40^{\circ}$ он составляет примерно $25^{\circ}$. Эксперименты, проведенные с использованием лазерных диодов с мощностью около $100 \mathrm{~mW}$ и длинами волн 0.65 и $0.53 \mu \mathrm{m}$, показали, что рассмотренный способ позволяет корректировать цвет изображений, в частности цвет лица человека.

Данный способ позволяет несколько изменять цвет изображений, восстанавливаемых монохромными голограммами, однако для получения полноцветных изображений необходимы запись голограмм и воспроизведение изображений на нескольких длинах волн. Особенности получения таких изображений при записи голограмм импульсным излучением будут рассмотрены в следующей работе.

\section{Заключение}

Установлены закономерности размерной трансформации голографических изображений протяженных по 
глубине объектов при изменении длин волн и геометрических параметров схем записи голограмм и восстановления изображений.

Предложен способ, позволяющий улучшать качество восстановленных изображений и снизить требования к выходным характеристикам импульсных лазеров.

Предложен аддитивный метод корректировки цвета изображений, восстанавливаемых монохромными пропускающими голограммами.

Результаты представляют интерес для решения задач импульсной голографии, связанных с регистрацией и восстановлением изображений протяженных объектов, в том числе изобразительной техники, а также выбора источников монохроматического излучения для компактных осветителей.

\section{Конфликт интересов}

Авторы заявляют, что у них нет конфликта интересов.

\section{Список литературы}

[1] Watanabe H., Okaichi N., Omura T., Kano M., Sasaki H., Kawakita M. // Sci. Rep. 2019. V. 9. P. 17688.

[2] Fan F., Yan X., Jiang X., Zhang T., Wen J, Su J., Yuan Q., Wang X., Song C., Han C. // Appl. Opt. 2019. V. 58. N 33. P. 9112.

[3] Zhang W., Sang X., Gao X., Yu X., Gao C., Yan B., Yu C. // Opt. Express. 2019. V. 27. N 22. P. 32810.

[4] Yan X., 1, Zhang T., Wang C., Fan F., Wang X., Wang Z., Bi J., Chen S., Lin M., Jiang X. // Opt. Express. 2019. V. 27. N 22. P. 32508.

[5] Askari M., Park J.-H. // Opt. Express. 2020. V. 28. N 1. P. 146.

[6] Su P., Gao Q.-X., He Z.-H., Ma J.-S. // Optik. 2020. V. 200. P. 163044.

[7] Choo H.-G., Kozacki T., Zaperty W., Chlipala M., Lim Y., Kim J. // Appl. Opt. 2019. V. 58. N 34. P. G96.

[8] Ito Y., Mitobe M., Nagahama M., Sakai H., Sakamoto Y. // Appl. Opt. 2019. V. 58. N 34. P. G135.

[9] Gentet Y., Gentet P. // Appl. Opt. 2019. V. 58. N 34. P. G226.

[10] Zhou H., Sui X., Cao L., Banerjee P.-P. // Appl. Opt. 2019. V. 58. N 34. P. G177.

[11] Vorzobova N.D., Staselko D.I. // Opt. Spectrosc. 1978. V. 45. N 1. P. 90.

[12] Артемьев Е.Ф., Беспалов В.Г., Брыскин В.З., Ворзобова Н.Д., Ермолаев М.М., Стаселько Д.И. // Оптическая голография. Практические применения. Л.: Наука, 1985. C. 107.

[13] Andreev R.B., Vorzobova N.D., Kalintsev A.G., Staselko D.I. // Opt. Spectrosc. 1980. V. 49. N 5. P. 514.

[14] Ворзобова Н.Д. // Оптическая голография. Практические применения. Л.: Наука, 1985. С. 65.

[15] Vorzobova N.D. // J. Opt. Technol. 2004. V. 71. N 10. P. 68.

[16] Ворзобова Н.Д. // Голография: фундаментальные исследования, инновационные проекты и нанотехнологии. Материалы XXVI школы по когерентной оптике и голографии. Иркутск: Папирус, 2008. С. 175.

[17] Vorzobova N.D., Ryabova R.V. // Proc. 10th International Symposium on Display Holography. 2015. P. 146-148.
[18] Collier R.J., Burckhardt C.B., Lin L.Y. Optical Holography. NY, London: Academic Press, 1973, 686 p.

[19] Джеймс T.X. Теория фотографического процесса. Л.: Химия, 1980, 672 с.

[20] Bjelkhagen H.J. Silver-halide recording materials for holography and their processing. New York: Springer Verlag, 1995. 